\title{
CARACTERIZACIÓN ENERGÉTICA DEL FUNCIONAMIENTO DE UN EQUIPO DE AIRE ACONDICIONADO EN UN LOCAL DADO.
}

\section{ENERGETIC CHARACTERIZATION OF THE FUNCTIONING OF AIR- CONDITIONING EQUIPMENT IN A GIVEN LOCALE}

\author{
MSc. Milen Balbis Morejón*, PhD. Francisco García Reina**, PhD. Juan José \\ Cabello Eras*, PhD. Valdimir Sousa Santos*. \\ *Institución 1, Facultad, Programa o Grupo de Investigación. \\ Dirección, Ciudad, Departamento, País. \\ Teléfono y Fax, con indicativos internacional y nacional. \\ E-mail: $\{$ autor1, autor3\}@dominio.edu.co. \\ ** Universidad de Ciego de Ávila (UNICA), Facultad de Ciencias Técnicas, Grupo de \\ Investigación en Energía. \\ Carretera de Morón Km 9, Ciego de Ávila, Cuba. \\ Teléfono: 53-33-225702, ext. 1060. \\ E-mail: pancho30011918@gmail.com.
}

Resumen: El control del consumo y eficiencia energética de los equipos de aire acondicionado es de vital importancia, sobre todo en lugares como la ciudad de Barranquilla con altas temperaturas y humedades relativas, lo cual genera en ésta un alto consumo de energía eléctrica. Se presenta en el siguiente trabajo un sistema de mediciones digitales que permite la caracterización energética completa del régimen de trabajo de un equipo de 2 toneladas de aire acondicionado en el enfriamiento y deshumidificación de un local de laboratorio de $98,7 \mathrm{~m} 3$ de volumen. Para esto se usan sensores digitales de temperatura y humedad relativa, con los cuales se registra el decremento de la temperatura y la humedad relativa en el tiempo. Con estos datos se calculan el calor sensible, el latente y la entalpía del aire húmedo que se extrajo de la habitación. Simultáneamente, se va registrando con un analizador de red digital el consumo de energía eléctrica por el equipo de aire acondicionado. Luego, el cociente entre calor sensible, el latente y la entalpía con la de energía eléctrica consumida da la eficiencia con que el equipo de aire acondicionado enfría el local (sensible), la eficiencia con que lo deshumidifica (latente) y la total respectivamente. Es importante destacar que este método de caracterizar y evaluar la eficiencia energética de aires acondicionados es aplicable a cualquier equipo y local que se quiera estudiar.

Palabras clave: aire acondicionado, eficiencia energética, calor latente, calor sensible, entalpía del aire húmedo.

\begin{abstract}
The control of the energy consumption and energetic efficiency of the airconditioning equipment is vitally important, mainly in location as Barranquilla's city with high temperatures and relative humidity, which generates herein high electric- energy consumption. Shows up in the following paper a digital- measurements system, which permits the complete energetic characterization of the work of a 2-tons equipment of airconditioning in the cooling and dehumidification of a laboratory of $98,7 \mathrm{~m}^{3}$ of volume. It is used digital temperature and relative humidity sensors for this measurement, with them as registers the decrement of the temperature and the relative humidity in the time itself.
\end{abstract}


The sensible and latent heats are calculated with these data, and the enthalpy extracted from the room. Simultaneously, it is registering the electric energy consumption for the air conditioning equipment, with a digital-net analyzer. Then, the quotient among sensible heat, the latent heat and the enthalpy with the electric energy consumed gives the efficiency that the air-conditioning equipment cools the locale, the efficiency with that the dehumidify and total efficiency, respectively.

Keywords: air conditioning, energetic efficiency, sensible heat, latent heat, wet air enthalpy.

\section{Introducción:}

El control del consumo y eficiencia energética de los equipos de aire acondicionado es de vital importancia, sobre todo en lugares como la ciudad de Barranquilla con altas temperaturas y humedades relativas, lo cual genera un alto consumo de energía eléctrica. Los sistemas de climatización son, en estos casos, de los mayores consumidores de energía eléctrica en instalaciones hoteleras, de oficinas, comerciales, industrias y domésticas (Zhenjun and Wand, 2009). Luego, para el uso eficiente de la energía eléctrica en esta tarea es de vital importancia contar con sistemas automatizados para el registro y control de este consumo energético. El problema es complejo en el sentido que los equipos de aire acondicionado (EAA) realmente realizan dos funciones simultáneamente: mantienen la temperatura por debajo de la del ambiente que rodea la habitación (la "enfrían") y además extraen vapor de agua del aire disminuyendo su humedad relativa (HR) (lo "secan") y en estos son los dos procesos principales donde debe de consumirse la energía eléctrica, en conjunto con los sistemas de ventilación para mover el aire por toda la habitación (Luck and Mago, 2012). En este trabajo se centra la atención en la determinación de la eficiencia energética con la que el EAA debe extraer calor de la habitación para bajar la temperatura (calor sensible) y la eficiencia con la que se extrae el vapor de agua (calor latente).

Para esto se desarrolló una tecnología de medición basada sobre sensores digitales de temperatura y HR para el registro continuo de ambas, así como el uso de un analizador de red digital para la medición del consumo de energía eléctrica, durante el proceso de arranque del EAA de llevar las condiciones del aire del local a las de confort (25 ${ }^{\circ} \mathrm{C}$ y $50 \%$ de HR), a partir de las condiciones iniciales de altas temperaturas y HR. Con el registro en el tiempo del decremento de la temperatura y la HR hasta llegar a las de confort, se calcula el calor extraído de la habitación para bajar la temperatura (sensible) y el extraído para baja la
HR (latente), usando para esto las ecuaciones de las propiedades termodinámicas del aire húmedo (Hyland and Wexler, 1998). La suma de estos valores da el decremento total de la entalpía del aire húmedo. Comparando ésta con el consumo de energía eléctrica por el EAA se determina la eficiencia energética del trabajo de este equipo en este local. Es interesante notar que esta metodología de caracterización energética del funcionamiento del EAA es válida para el estudio de cualquier equipo en cualquier habitación (Luck and Mago, 2012).

\section{Fundamentación teórica.}

Las propiedades termodinámicas del aire húmedo han sido objeto de un minucioso estudio desde mediados del siglo pasado, debido a su fundamental importancia para el diseño y construcción de sistemas de aclimatación de locales, tanto si es necesario enfriarlos en climas tropicales como si es necesario calentarlos en climas fríos (Zhenjun and Wand, 2009). En la literatura se reportan muchos excelentes trabajos con las tablas y ecuaciones de ajuste para los valores de todas las propiedades termodinámicas de la mezcla de aire seco con vapor de agua: (Greenspan, 1976) (Davies, 1992) (Melling et al., 1997) (Tsilingiris, 2009). Para este trabajo se usarán las ecuaciones y tablas dadas en el Handbook de la ASHRAE (2009), por su novedad, actualización y rigor.

La entalpía específica del aire seco está dada por:

$$
h_{a s}=c_{p_{a s}} \cdot t
$$

en $\mathrm{J} / \mathrm{kg}$, donde $c_{a s}$ es el calor específico del aire seco $(1005 \mathrm{~J} / \mathrm{kgK})$ y t es la temperatura absoluta. La entalpía del vapor de agua es:

$$
h_{v}=X . c_{p_{v}} \cdot t+X . L_{f}
$$

donde $X$ es la masa en $\mathrm{kg}$ de vapor de agua, $c_{p v}=$ $1872,3 \mathrm{~J} / \mathrm{kgK}$ es el calor específico del vapor de 
agua y $L_{f}=2,257 \mathrm{MJ} / \mathrm{kg}$ es el calor latente de vaporización del agua.

Luego la entalpía específica para el aire húmedo sería igual a la entalpía para $1 \mathrm{~kg}$ de aire seco más la del vapor de agua (Tsilingiris, 2009):

$$
h=c_{p_{a s}} \cdot t+\left(c_{p_{v}} \cdot t+L_{f}\right) X
$$

Sustituyendo los valores de las constantes y agrupando los términos dependientes de la temperatura y la masa:

$$
h=(1005+1872,3) t+2257.10^{3} . X_{(4)}
$$

la cual da la entalpía del aire húmedo en $\mathrm{J} / \mathrm{kg}$, y que realmente significa la cantidad de calor que hay que extraer al aire húmedo para bajar la temperatura desde el valor inicial $t$ hasta el deseado y la masa de vapor de agua en el aire $X$ hasta el valor de la HR deseada. El primer término, que solo depende de la temperatura, el cual se puede sentir es el llamado "calor sensible", mientras que el segundo que es el que depende de la masa de vapor de agua se le llama "calor latente".

Para determinar la masa de vapor de agua $X$ en el aire es necesario realizar el cálculo psicrométrico según el Handbook de la ASHRAE (2009) y (Luck and Mago, 2012). La presión de vapor de agua saturado en el aire $P_{w s}$ está dada por:

$$
P w s=\exp \left(\frac{C_{1}}{T}+C_{2}+C_{3} T+C_{4} T^{2}+C_{5} T^{3}+C_{6} \ln (T)\right)
$$

donde $\mathrm{T}$ es la temperatura absoluta y las constantes $\mathrm{C}_{1}-\mathrm{C}_{6}$ son:

$$
\begin{array}{lll}
C_{1}=-5,800.2206 & C_{2}=1.3914993 & C_{3}-0.048640239 \\
C_{4}=4.1764769 \times 10^{-5} & C_{5}=-1.4452093 \times 10^{-8} & C_{6}=6.5459673
\end{array}
$$

en esta ecuación la temperatura se da en $\mathrm{K}$ y la $P_{w s}$ en $\mathrm{kPa}$.

La HR se determina por:

$$
\emptyset=\frac{P w}{P w s}
$$

donde $P_{w}$ es la presión parcial del vapor de gua en el aire. La razón de humedad en $\mathrm{kg}$ de vapor de agua por $\mathrm{kg}$ de aire seco viene dada por:

$$
\omega(\mathrm{T}, \phi):=\frac{18}{29} \cdot \frac{\phi \mathrm{p}_{\mathrm{ws}}(\mathrm{T})}{\mathrm{p}_{\mathrm{a}}-\phi \mathrm{p}_{\mathrm{ws}}(\mathrm{T})}
$$

donde $p_{a}$ es la presión atmosférica. Como que la densidad del aire seco varía muy poco en el rango de temperaturas de 20 a $40{ }^{\circ} \mathrm{C}$ (Tsilingiris, 2008) se puede tomar un valor medio de $\rho_{\text {air }}=1,125 \mathrm{~kg} / \mathrm{m}^{3}$. Luego multiplicando la ecuación anterior por este valor de la densidad del aire seco se obtiene la masa de agua por $\mathrm{m}^{3}$ de aire en función de la temperatura y la HR para evaluar entonces la entalpía del aire húmedo. Esto es muy útil en esta forma, ya que muchas veces lo que se conoce es el volumen de la habitación.

\section{Materiales y métodos.}

En la figura 1 se presenta el sensor digital de temperatura y HR de la firma alemana TESTO Saveris 2, el cual registra la temperatura ambiente con un error de $\pm 0,5^{\circ} \mathrm{C}$ y la $\mathrm{HR}$ con un error de \pm $2,0 \%$. Tiene conexión inalámbrica a $\mathrm{PC}$ para el registro continuo y automático de las mediciones en el tiempo.

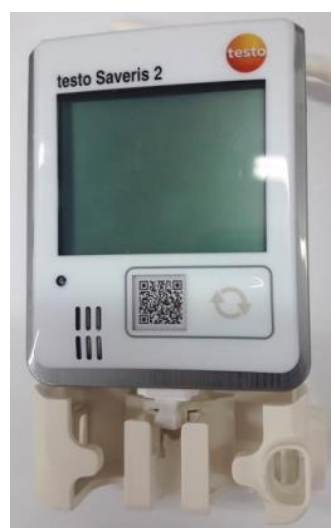

Figura 1: Sensor de temperatura y HR TESTO.

En la figura 2 se presenta el analizador de red para la medición del consumo de energía eléctrica de la firma SIEMENS (Eslovenia), marca METREL. Es digital y guarda en memoria acoplada a PC el resultado de las mediciones. El error en la medicón de potencia y energía eléctrica es menor del $1 \%$. El equipo de aire acondicionado es de la firma REFRINORTE en Barranquilla, Colombia y es de 2 Ton. El local del laboratorio es interno sin parades expuestas al sol, sin ventanas y de un volumen de $98,72 \mathrm{~m}^{3}$. 


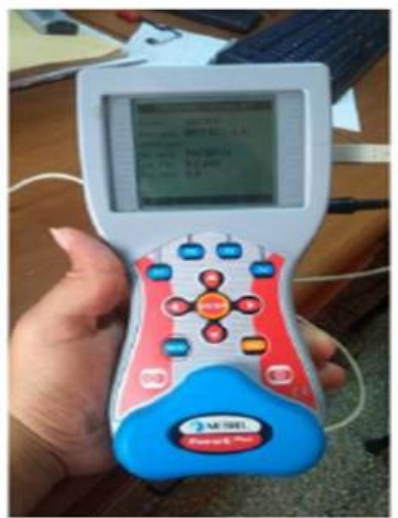

Figura 2: Analizador de red marca METREL.

\section{Resultados y su discusión:}

En la figura 3 se presenta la variación en el tiempo de los valores medios de temperatura y HR desde los valores iniciales hasta llegar a los valores de confort de $25^{\circ} \mathrm{C}$ y $50 \%$ de HR, respectivamente. Como era de esperar, es aproximadamente lineal en ambos casos (Zhejun and Wang, 2009).

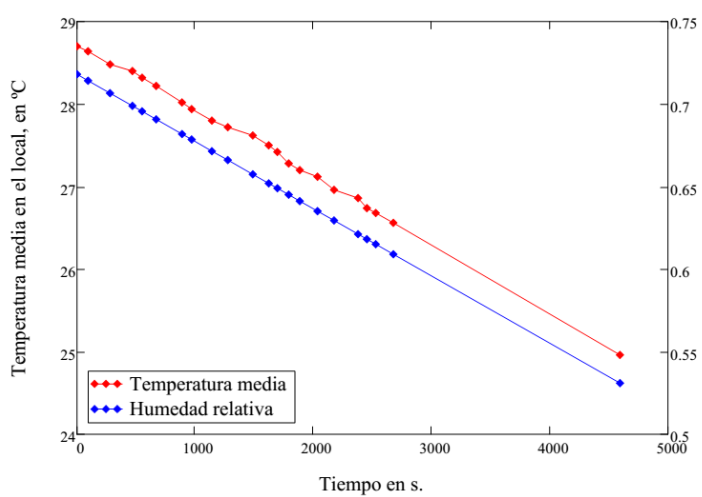

Figura 3. Temperatura y HR de la habitación en el tiempo.

Restando los valores obtenidos de $\mathrm{T}$ y HR del valor inicial se ontienen los decrementos de ambas, como se muestra en la figura 4.

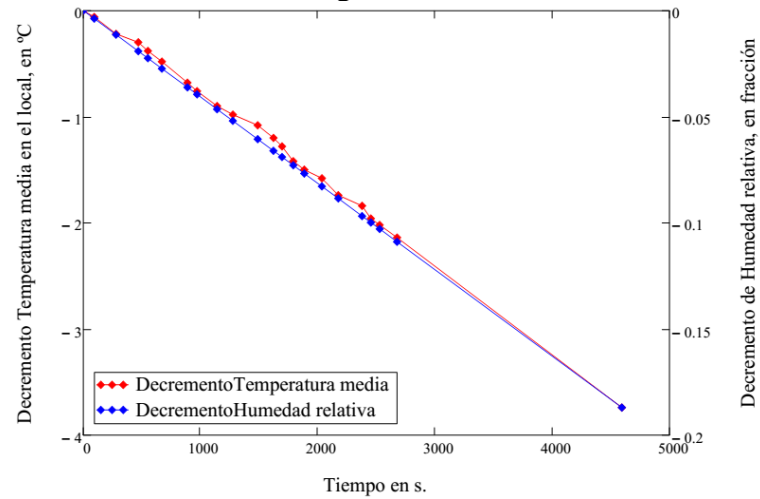

Figura 4. Decremento de la temperatura y HR de la habitación en el tiempo.
Con estos datos se calcula la masa de agua en el aire en $\mathrm{kg}$ por $\mathrm{kg}$ de aire seco, como se muestra en la figura 5 por la ecuación (7):

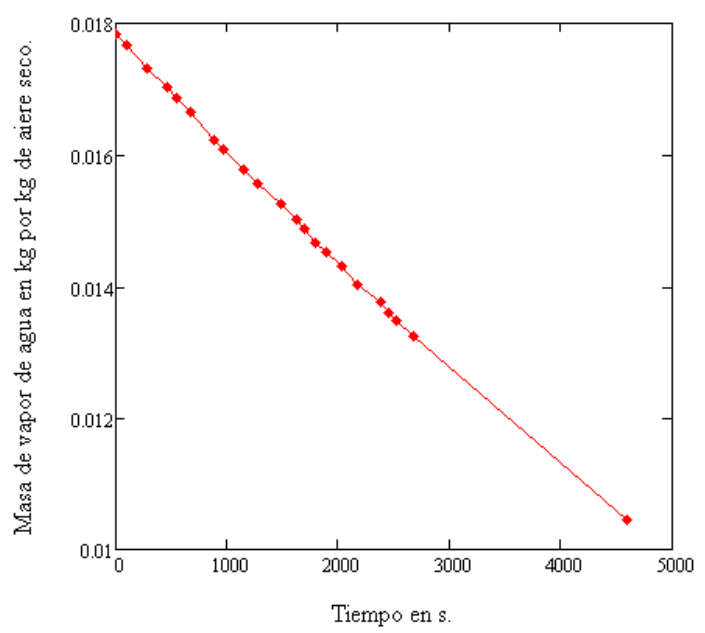

Figura 5. Masa de agua en el aire en $\mathrm{kg}$ por $\mathrm{kg}$ de aire seco.

$\mathrm{O}$ teniendo en cuenta que en $1 \mathrm{~m}^{3}$ de aire seco hay una masa de $1,125 \mathrm{~kg}$ se calcula la masa de vapor de agua por $\mathrm{m}^{3}$, como se muestra en la figura 6 , la cual es negativa por ser una masa extraída al sistema:

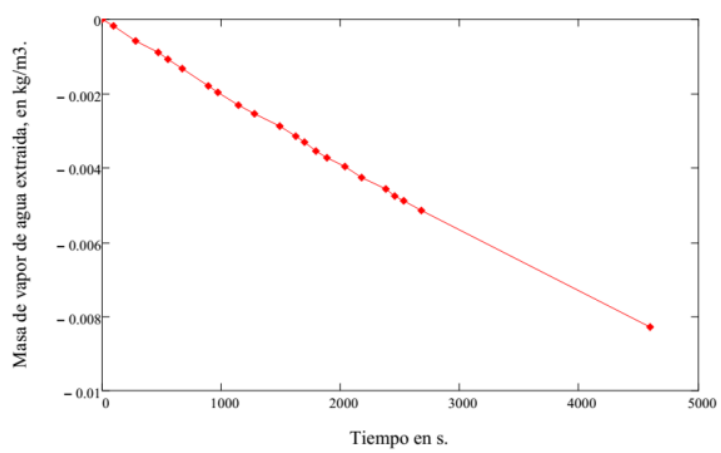

Figura 5. Masa de vapor de agua extraída por $\mathrm{m}^{3} \mathrm{de}$ aire.

Usando la ecuación (4) se determina el calor sensible y el latente extraído del aire para bajar la temperatura y la HR por cada $\mathrm{m}^{3}$, como se muestra en la figura 6. 


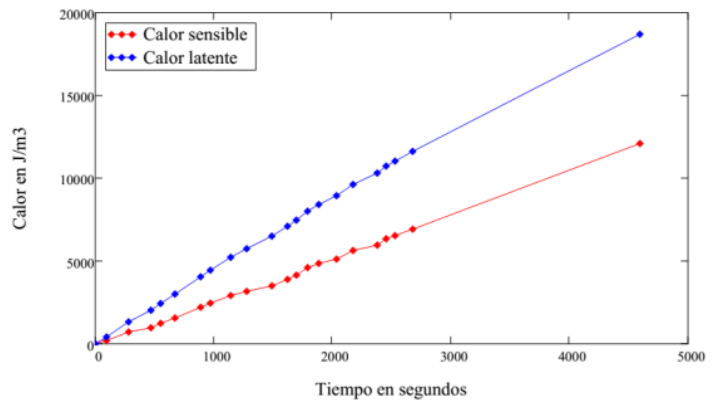

Figura 6. Calor sensible y el latente extraído del aire para bajar la temperatura y la HR por cada $\mathrm{m}^{3}$ de aire.

Como puede apreciarse el calor extraído para condensar el agua (latente) es mayor siempre que el extraído para bajar la temperatura (sensible). La suma de ambos es la variación total de entalpía que experimentó el aire húmedo en este proceso (figura 7).

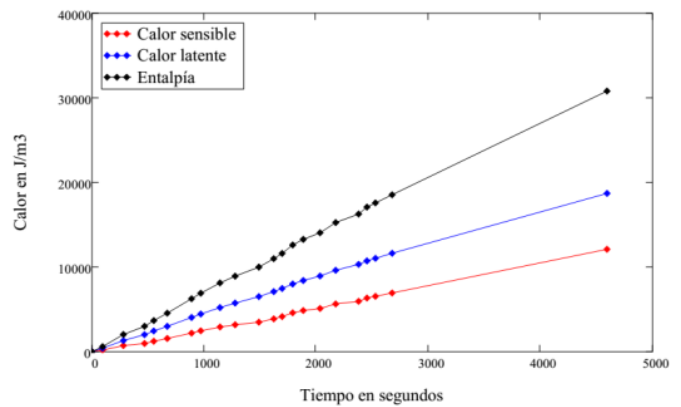

Figura 7. Calor sensible, latente y entalpía por $\mathrm{m}^{3}$.

y teniendo en cuenta que el volumen de la habitación es de $98,72 \mathrm{~m}^{3}$ se tiene para los valores totales de calor sensible, latente y entalpía los valores de la figura 8 .

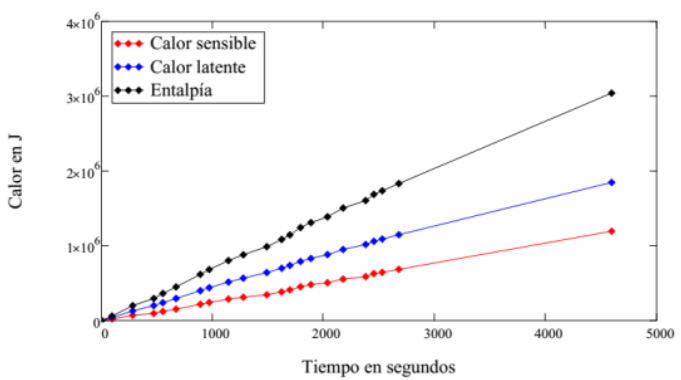

Figura 8. Valores totales del calor sensible, latente y entalpía

En la figura 9 se presenta además de los valores anteriores de calor sensible, latente y entalpía, el consumo de energía eléctrica acumulado por el EAA en este período de tiempo.

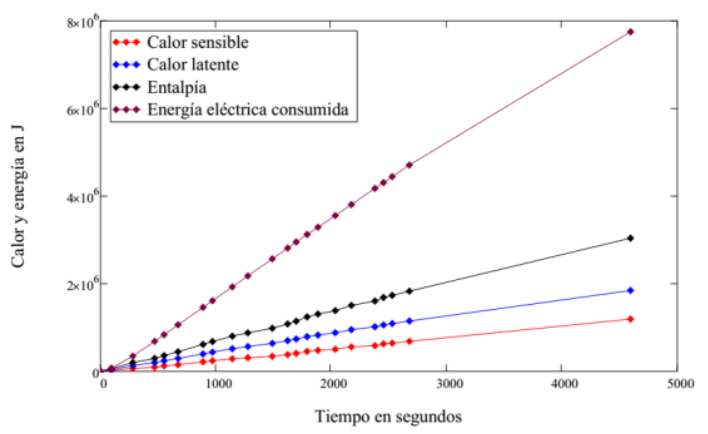

Figura 9. Valores totales del calor sensible, latente, entalpía y energía eléctrica consumida.

La energía eléctrica consumida es más del doble que la variación total de entalpía, ya que el EAA no tan solo enfría y deshumidifica al aire, sino que también tiene que moverlo, es decir en esta medición no se tuvo en cuenta la energía eléctrica consumida por los ventiladores del EAA, lo cual será objeto de otra investigación.

El cociente entre los valores totales del calor sensible, latente y entalpía con la energía eléctrica consumida (multiplicado por 100) da la eficiencia energética de cada uno de estos procesos, con relación a la energía total consumida por el equipo, como se muestra en la figura 10.

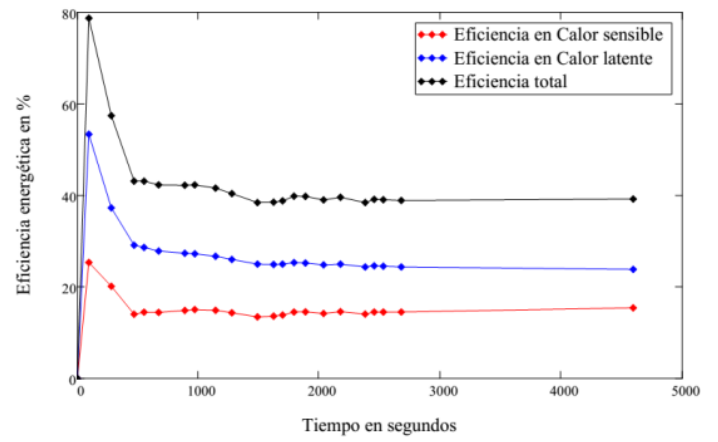

Figura 10. Eficiencia energética en $\%$ de los procesos de enfriado, secado del aire y la total en la habitación por el EAA.

Como puede apreciarse el sistema de variador de frecuencia del EAA hace que el inicio no haya un consumo excesivo de energía eléctrica por lo que la eficiencia del proceso es alta. Por supuesto, a medida que se estabiliza el proceso el consumo de energía por los ventiladores se incrementa en comparación con el de enfriado y secado, por lo que la eficiencia disminuye (Zhenjun and Wang, 2009).

\section{Conclusiones}

Esta metodología para la caracterización y evaluación energética de los equipos de aire 
acondicionado permite evaluar el funcionamiento de los mismos de forma rápida, segura y rigurosa, para el uso eficiente de la energía energía eléctrica. $\mathrm{Se}$ ha venido usando con éxito en diferentes aplicaciones, pero la de mayor incidencia ha sido en la evalución del efecto del mantenimiento y limpieza de los EAA y en la determinación eficaz del ciclo de estos.

\section{Bibliografía:}

ASHRAE. (2009). Handbook - Fundamentals (SI Edition), American Society of Heating, Refrigerating and Air Conditioning Engineers, Inc., Atlanta, GA.

Davies RS. (1992).Equation for the determination of density of moist air. Metrologia; 29:67-70.

Greenspan L. (1976). Functional equations for the enhancement factors for $\mathrm{CO} 2$-free moist air. $\mathrm{J}$ Res National Bureau Standards-A Phys Chem; 80A(1):41-44.

Hyland RW, Wexler A. (1983). Formulations for the thermodynamic properties of dry air from $173.15 \mathrm{~K}$ to $473.15 \mathrm{~K}$ and of saturated moist air from 173.15 to $372.15 \mathrm{~K}$ at pressures to 5 MPa. Trans ASHRAE;89(IIa):520-535.

Luck R. and Mago P. J. (2012). Use of MathCad and Excel to Enhance the Study of Psychrometric Processes for Buildings in an Air Conditioning Course. ASEE Southeast Section Conference. Mississippi State University. MI, USA.

Melling A, Noppenberger S, Still M, Venzke H. (1997). Interpolation correlations for fluid properties of humid air in the temperature range $100{ }^{\circ} \mathrm{C}$ to $200{ }^{\circ} \mathrm{C}$. J Phys Chem Ref Data 1997; 26(4):1111-1123.

P.T. Tsilingiris. (2008). Thermophysical and transport properties of humid air at temperature range between 0 and $100{ }^{\circ} \mathrm{C}$. Energy Conversion and Management 49 1098-1110.

Zhenjun M. and Shengwei Wang. (2009). Energy efficient control of variable speed pumps in complex building central air-conditioning systems. Energy and Buildings 41 197-205. 